\title{
Atrial conduction pathways
}

\author{
Anurag Singh MD, Kenneth Nugent MD
}

In this issue of the Southwest Respiratory and Critical Care Chronicles, Argueta and coworkers report a patient who underwent two surgical procedures to resect a recurrent left atrial myxoma. After the second procedure, she developed cardiac arrhythmias and $\mathrm{AV}$ conduction blocks and eventually required a dual-chamber pacemaker. The pathways relevant to this outcome are illustrated in the Figure below. ${ }^{1}$

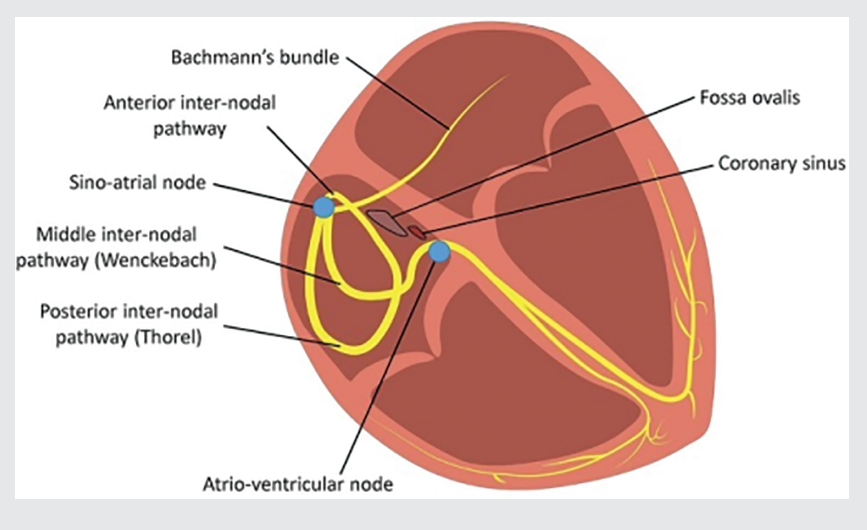

Electric impulses are generated in the sinoatrial node (located superiorly in the right atrium at the junction with the superior vena cava) and the conduction of depolarization waves from the sinoatrial node across the right atrium to the atrioventricular node occurs through three internodal tracts, the anterior, the middle, and the posterior tracts. ${ }^{2}$ The anterior tract, in addition, gives rise to a trapezoid shaped bundle of fibers which course along the superior quadrant of the interatrial sulcus from the right atrium into the left atrium. This bundle is called the Bachmann's bundle and the conduction of electrical impulses from the

Corresponding author: Anurag Singh

Contact Information: Anurag.singh@ttuhsc.edu

DOI: 10.12746/swrccc.v6i25.485 right atrium to the left atrium occurs through this tract. During sinus rhythm, the electrical impulses originate in the right atrium and move to the left atrium predominantly across the Bachmann's bundle enabling almost simultaneous depolarization of both the right and left atria to maintain synchrony. The right coronary artery usually supplies the sinoatrial node and Bachmann's bundle.

Conduction delays in this tract cause an interatrial block and prolonged P-waves (>110 msec). These $P$ waves are often notched signifying delayed depolarization of the left atrium and in case of any block in this bundle, electrical forces then move from the right atrium to the atrioventricular node and then via fibers in the coronary sinus move into the left atrium causing depolarization in the left atrium to start inferiorly and progress superiorly. This causes biphasic $P$ waves as the two atria depolarize in opposite directions. Delays in Bachmann's bundle can cause supraventricular arrhythmias, such as atrial tachycardia and/or fibrillation arising from the left atrium, and asynchronous contraction of the right and left atrium. These arrhythmias and delays ultimately lead to left atrial remodeling manifesting as left atrial enlargement.

Surgical procedures involving the interatrial septum can injure these pathways and therefore lead to loss of interatrial synchrony. In particular, surgical resection of left atrial myxomas in an atypical location, such as near the sinus node, can injure the sinus node and the Bachmann's bundle. ${ }^{3}$ This results in abnormal impulses arising from nonsinus node locations in the right and left atrium leading to arrhythmias, like atrial tachycardia and atrial flutter/fibrillation. Consequently, surgeons must pay close attention to the location and anatomy of cardiac tumors to avoid damage to these normal electrical conduction pathways. It is therefore advised to obtain pre and post-operative ECGs to detect conduction delays and monitor for arrhythmias during the immediate postoperative period so that 
early diagnosis can be made and appropriate steps taken during the postoperative recovery period.

Keywords: Bachmann's bundle, interatrial conduction, atrial myxomas, surgery

From: The Department of Internal Medicine at Texas Tech University Health Sciences Center in Lubbock, Texas

Submitted: $7 / 4 / 2018$

Conflicts of interest: none

This work is licensed under a Creative Commons Attribution-ShareAlike 4.0 International License

\section{REFERENCES}

1. Tse G, Lai ET, Yeo JM, Yan BP. Electrophysiological mechanisms of Bayés syndrome: insights from clinical and mouse studies. Front Physiol 2016;7:188. Published online 2016 May 31. doi: 10.3389/fphys.2016.00188. This figure was downloaded from OPENi(beta), an open source media repository, on the Texas Tech University Health Sciences Center Library website on 7/3/2018.

2. Ariyarajah V Spodick DH. The Bachmann bundle and interatrial conduction. Cardiology Review 2006;14:194-199.

3. Hatz DE, Danielson D, Young JN, et al. The impact of preoperative imaging for atypical atrial myxoma locations. Clinical Cardiology 2012;35:490-493. 